\title{
Deconvolution of interferometric observations by MIM
}

\section{J. Pfleiderer and G. Köb}

Institut für Astronomie der Leopold-Franzens-Universität Innsbruck, Technikerstraße 25, A-6020 Innsbruck, Austria

Received May 23; accepted October 06, 1997

\begin{abstract}
We derive deconvolution equations - dirty map and dirty beam - without use of Fourier transforms. We thus avoid interpolation of observed complex visibilities $(\mathrm{CVs})$ as well as aliasing effects. MIM (= minimum information method) implies the modification of the dirty beam such that a straightforward deconvolution without constraints gives a smoothed result. Smoothing can be diminished at edges and point sources by modification of the dirty map near these features. We give test examples in which we discuss in detail the Gibbs phenomenon encountered in any smoothed deconvolution of sharp features. Finally, we deconvolve old VLBI observations of 3C 309.1.
\end{abstract}

Key words: methods: data analysis - techniques: image processing, interferometric - quasars: individual: 3C 309.1

\section{Introduction}

This paper elaborates ideas presented in 1992 by Kus et al. It deconvolves interferometric data without explicit use of discrete Fourier transforms (DFT) between image space and visibility space (frequency space) because such transformations need an equidistant rectangular grid on both sides. The grids must be large enough to avoid aliasing effects. To the contrary, an observational grid of complex visibilities $(\mathrm{CVs})$ is, generally, neither equidistant nor rectangular nor sufficiently large.

Usually, the observed CVs in the $u, v$-plane are interpolated onto a rectangular equidistant grid. Where no observations exist, zero values of the corresponding $\mathrm{CVs}$ are assumed. The real part of the DFT of those interpolated and extended CVs is called the dirty map (DM). The image, or Clean Map (CM), is found from the dirty one by deconvolution with the dirty beam (DB). Several deconvolution methods and algorithms are available. All the usual ones work with DFTs, i.e., do the convolution $D M=C M \otimes D B$ by DFT. One can easily recognize flaws in this standard procedure. The first is introduced by the CV interpolation. In particular, interpolation into an equidistant grid, i.e. constant $\mathrm{CV}$ density in the $u, v$ plane, means that the measured CVs are weighted according to the reciprocal $\mathrm{CV}$ density. Second, the use of DFTs between image space and visibility space implies periodicity of the maps and thus creates aliasing effects. One can as well say that CM, DB, and DM are assumed to be connected by a Fourier convolution instead of a straight convolution.

We have devised a method to avoid these flaws. The Minimum Information Method MIM (Pfleiderer 1985) does a Least-Squares-Fit (LSF) of observed data. That is to the effect that we make use of the so-called direct Fourier transform which is well known in imaging (Sramek \& Schwab 1986) but not in deconvolution.

The deconvolution equations are considered as a linear system of equations connecting the maps DM, DB, and CM, which is to be solved only approximately within the error tolerances. Overfitting is avoided by a diophantic solution of the error normalized equations. The method of solution is similar but not identical to CLEAN. Smoothing is accomplished by a local smoothing constraint (Pfleiderer 1989) which changes the DB into a Prussian Helmet type beam. The linear connection between CM and DM is, however, kept. The procedure is comparable to but not identical with the "Smoothness stabilized CLEAN" (Cornwell 1983). Our formalism is given in Sect. 2, the deconvolution algorithm in Sect. 3, the detection and deconvolution of steep features in Sect. 4, some tests in Sect. 5, and our deconvolution of old VLBI data of 3C 309.1 in Sect. 6.

\section{Deconvolution equations}

The observed CVs have well-known spatial frequencies (frequency pairs in the usual twodimensional case) and contain unknown complex-amplitude errors. The visibilities result from a Fourier integral over the intensity distribution in the image space. For practical purposes, the continuous intensity distribution has to be approximated by a discrete intensity grid. While it is not strictly necessary, it is nevertheless convenient, also for practical purposes, to consider a rectangular equidistant grid of constant-size intensity pixels. 
Thus, we start with such grid and intensities $z_{j l}$ at grid positions $x_{j l}=\left\{x_{j}=j \Delta x\right.$ and $\left.y_{l}=l \Delta y\right\}$. Given an observed frequency pair $\left(u_{k}, v_{k}\right)$, the observed CV (complex amplitude $A_{k}$ ) is given by

$$
\left(A_{k}-\epsilon_{k}\right) f_{k}=S_{k, j l} \sum_{j} \sum_{l} z_{j l} \mathrm{e}^{-2 \pi i\left(u_{k} x_{j}+v_{k} y_{l}\right)}
$$

where $f_{k}$ is a - generally known - telescope-dependent sensitivity factor which we shall put, for convenience, as equal to $1 . \epsilon_{k}$ is a complex error. The $z_{j l}$ are the unknowns to be found from the observations. Thus, we call the righthand side of Eq. (1) the visibility model $\left\{M_{k}\right\}, k=1, K$.

Another sensitivity factor $S_{k, j l}$ is included to take into account the influence of the intensity distribution within the pixel $j l$ on the observations. It is not known observationally but some assumption has to be made, at least implicitly. For a model, it is useful to assume the same distribution in all pixels: then $S$ depends only on frequency, $S=S_{k}$. For example, $S_{k}=1$ if the intensities were concentrated in the mid-pixel positions $\left\{x_{j}, y_{l}\right\}$. If, instead, one fourth of the intensity is put into each of the four pixel corners $x_{j} \pm \Delta x / 2, \quad y_{l} \pm \Delta y / 2$ then $S_{k}=\cos \left(\pi u_{k} \Delta x\right) \cos \left(\pi v_{k} \Delta y\right)$. A non-symmetric intensity distribution within the pixel would give rise to a complex $S_{k}$. If each pixel has constant surface brightness $\beta_{j l}=z_{j l} / \Delta x \Delta y$ then

$S_{k}=\operatorname{sinc}\left(u_{k} \Delta x\right) \operatorname{sinc}\left(v_{k} \Delta y\right)$

which is what we adopt in the following. The reason is that the influence of the pixel size chosen on the deconvolution result is diminished. In any case, $S_{k}$ is near unity for small frequencies and/or small pixel size because, then, the exponential factor varies little within each pixel.

Note that each CV is a member of a DFT of the image $\{z\}$ but, unless the frequency pairs are given on a rectangular grid, each CV is a member of a different DFT. Interchanging the role of the two telescopes giving rise to a certain $\mathrm{CV}$ changes the sign of the frequencies. Both CVs are, of course, equivalent, and either may be used. Thus, $A_{-k}=A_{k}^{*}$ and $\epsilon_{-k}=\epsilon_{k}^{*}$.

The CVs being a linear superposition of (weighted) intensities (which are the unknowns), an LSF is apt. The LSF weights $w_{k}=1 / \sigma_{k}^{2}$ can be understood in the sense that one expects to reproduce the corresponding term within an error that can be considered as a member of an (approximately) Gaussian distribution with variance $\sigma_{k}^{2}$. In principle, one can choose the $\sigma$ freely, e.g. according to natural weighting, or to uniform weighting. It would, however, not be useful to choose them smaller than the actual measuring errors. In other words, the choice should be $\sigma_{k}^{2} \geq E\left(\epsilon_{k} \epsilon_{k}^{*}\right)$ where of course the quadratic error expectation $E$ is no more known than the actual error $\epsilon_{k}$ but can often be reasonably estimated. In MIM, the equations are normalized to a unit error expectation $(0,1)$, that is, zero average and unit variance. Also, the intensity units can be normalized to multiples of the point source detection limit

$z_{0}=\frac{1}{\sqrt{\sum_{k} \frac{1}{\sigma_{k}^{2}} S_{k}^{2}}}$.

We keep the errors formally in the equations and minimize the absolute value of model minus observed $\mathrm{CVs}$,

$\sum_{k} \frac{1}{\sigma_{k}^{2}}\left(M_{k}+\epsilon_{k}-A_{k}\right)\left(M_{-k}+\epsilon_{-k}-A_{-k}\right)=\min$.

The result of differentiation in respect to $z_{n m}$ is

$\sum_{j} \sum_{l} z_{j l}^{*} a_{j-n, l-m}-b_{n m}=: r_{n m}$

with $z^{*}=z / z_{0}$

$a_{j-n, l-m}=$

$=z_{0}^{2} \sum_{k} \frac{1}{\sigma_{k}^{2}} S_{k}^{2} \cos \left[2 \pi\left(u_{k}\left(x_{j}-x_{n}\right)+v_{k}\left(y_{l}-y_{m}\right)\right)\right]$

$a_{0,0}=1 \quad$ and $\quad\left|a_{i, k}\right|<1 \quad[i \neq 0$ or $k \neq 0]$

$b_{n m}=z_{0} \sum_{k} \frac{1}{\sigma_{k}^{2}} S_{k} \Re\left(A_{k} \mathrm{e}^{\left.2 \pi i\left(u_{k} x_{n}+v_{k} y_{m}\right)\right)}\right.$.

The system is to be solved in the sense that the residuals $\left\{r_{n m}\right\}$ fit approximately into a $(0,1)$ distribution. The double sum is, apparently, a discrete convolution (DC) of the intensity map $z^{*}$ with a dirty beam $a$, i.e., we have a straight deconvolution problem, not a Fourier deconvolution problem.

It is convenient to put $\sigma_{k}=\sigma=$ const. Then $z_{0}=$ $\sigma / \sqrt{\sum_{k} S_{k}^{2}}$. Each term of the dirty map $b$ is the real part of one (weighted) term of a DFT of the CVs. But the DM as a whole is the real part of such DFT only if the CVs are given on a rectangular frequency grid (and complemented by zeros where missing). The same holds for the DB. In this case, $a$ and $b$ would, except for the weighting, just be the DB and DM of the usual procedure. Of course, the pixels of the image $z$ would have to be defined on a corresponding rectangular grid; in particular, the pixel number has to equal the number of (complemented) CVs, and each Fourier term has to have equal weight. To the contrary, our DM and DB, i.e., $b$ and $a$, can be considered as a (weighted) direct Fourier transform (see Sramek \& Schwab 1986) of the CVs and the corresponding unit numbers using both signs of the frequencies. Note that, inspite of its name, this transformation is not a Fourier transform in the usual sense. Unless certain conditions are met, not even a unique back transformation is possible.

We can thus freely choose a common pixel size for CM, $\mathrm{DM}$, and DB, as well as the number of pixels. Of course, a reasonable pixel size is approximately given by the special problem under consideration. The central beam should not be undersampled while there is little advantage in oversampling it. 
The size of the image need not be larger than what is needed to contain all expected intensity. The DB should be large enough to allow a full convolution with the CM in the range of the DM. It should be noted that none of the 3 maps is periodic or assumed to be so. Even if the CVs were given on an rectangular grid, there would be no periodicity.

The overall goodness of the fit can be checked by the sum of the squared residual visibilities. We find

$$
\begin{aligned}
R: & =\sum_{k} \frac{1}{\sigma_{k}^{2}}\left(A_{k}-M_{k}\right)\left(A_{-k}-M_{-k}\right)= \\
& =\sum_{k} \frac{1}{\sigma_{k}^{2}} A_{k} A_{-k}-\sum_{i} \sum_{j} z_{i j}^{*}\left(b_{i j}-r_{i j}\right)
\end{aligned}
$$

\section{Method of solution}

We consider Eq. (5) as a linear system of equations which is to be solved not exactly but only within the $(0,1)$ error distributions. The justification for doing so within an LSF is that we do not aim at minimizing the residuals, that is, the remaining error, but rather aim at minimizing the deviations from a model that leaves a set of expected residuals. Also, an exact LSF would ask for less variables than data, i.e., for a sufficiently small image.

Of course, there are many solutions within the error expectance, and this is where constraints come in. The first one is (in case of intensities) non-negativeness (which we take into account during the solving algorithm), the second, more important one is smoothness of the image.

Most smoothing constraints, as maximum entropy or minimum second derivative, are global in the sense that smoothing at one place may affect the solution at other places. Suppression of higher spatial frequencies is also of this kind. Our constraint is local. It is derived from the concept of structural information (Pfleiderer 1989) which is minimized. Here, we only need mention that it is built from squared intensity differences. Its derivative which is used in the minimization process is thus linear in the intensities. Added as a Lagrange condition to the system of Eq. (4), it produces again a linear system with modified coefficients. Instead of solving Eq. (5) under a constraint, i.e., deconvolving the DM with the true DB (the $a$ in Eq. (5)) but allowing for the constraint, we deconvolve the DM without constraint but with a modified DB.

The modification consists of adding a "smoothing beam" $s$ to the coefficients, $\tilde{a}_{i j}=a_{i j}+\lambda s_{i j}$ where $\lambda$ is a Lagrange parameter which is not fixed by theory but can be freely chosen. The larger $\lambda$, the more smoothing occurs. The choice $\lambda=1$ is generally a good one. The function $s$ is zero outside the main beam (to the effect that we do not smooth over a larger area), is negative inside the main beam with increasing values towards the center and the sum equalling -1 , and is positive $=+1$ in the center of the main beam $(\Sigma s=0)$. That is, $a_{00}$ is replaced by $\tilde{a}_{00}=1+\lambda$, surrounded by $\tilde{a}<a$. Thus $\tilde{a}_{00}$ protrudes from the surrounding beam. This spike gave rise to the name Prussian Helmet type beam.

The smoothing action works as follows: If a point source is somewhere introduced, i.e., the beam is subtracted from the dirty map, then the remaining residuals are too small in the center, and too large in the surroundings. That is, further intensity has to be put into the surrounding area in order to achieve an overall fit.

The exact definition of $s$ is not very critical. The theory leaves it open. The more the negative $s$ is concentrated towards the center, the smaller is the region over which smoothing takes place. The whole procedure has been described in some detail by Pfleiderer $(1985,1988)$.

Cornwell (1983) used a similar approach in his socalled Smoothness-Stabilized CLEAN. He minimized the sum of squared intensities which is equivalent to putting $s_{00}=1$ and $s=0$ everywhere else (i.e., $\Sigma s \neq 0$ ). He was probably the first to use a Prussian Helmet beam.

The normalization of Eq. (5) with expected residuals within a $(0,1)$ error distribution, together with $|a| \leq$ 1 , has the advantage that it suffices to determine the $z^{*}$ to within about \pm 1 . That is, it suffices to determine a diophantic solution. Thus, overfitting below the expected noise level is avoided.

We find the solution by a method comparable to the iterative CLEAN where the maximum of the DM is found and a point source is subtracted from it, the intensity of which is determined by the gain factor. The simplest way to solve our system is: Find the maximum of the DM, subtract the beam $\tilde{a}$ corresponding to a point source of intensity 1 (i.e., intensity = point source detection limit $\left.z_{0}\right)$, and iterate. Subtract a point source of intensity -1 if the minimum of the DM is sufficiently negative, and if the resulting intensity at this point stays non-negative. We have used (with slight modifications) this rather primitive algorithm because a better method to solve a linear system of equations under the constraints that the solution be diophantic and non-negative is not known to us. Of course, the final smoothing of CLEAN over the main beam is not necessary in our case.

\section{Edge detection}

All smoothing deconvolutions show Gibbs phenomena ("ringing") near steep slopes, as point sources or edges, which are smeared out over the smoothing area. The data are not fitted well near such places. E.g., a smoothed point source has too little intensity in the center, and too much around. This overshooting of intensity in the vicinity of the point source is compensated by undershooting farther out, and this again by overshooting still farther out, etc.

In order to reduce such ringing, it is necessary to deconvolve the corresponding steep features with reduced smoothing. One possibility is Two Channel Restoration (Gratl 1998) in which steep features, especially point sources, are recognized and their convolution with the DB 
subtracted from the DM, followed by the deconvolution proper which can include smoothing because the steep features have been removed. The method permits subpixel accuracy and can be used with any deconvolution method.

MIM offers another possibility because it uses two different beams, $a$ and $s$. Smoothing can be switched off locally by putting $\lambda=0$ locally (Pfleiderer 1985). We at present prefer to change the DM such that the code will consider edges and point sources formally as being smooth. Subpixel accuracy is not possible.

The dirty map DM (errors neglected) is a convolution of the unknown true map TM with the dirty beam $a$,

$a \otimes T M=D M$

which is equivalent to

$(a+\lambda s) \otimes T M=D M+\lambda s \otimes T M$.

By adding the true map convolved with the smoothing beam to the dirty map, one can deconvolve with the smoothness-modified beam $a+\lambda s$ and still recover the edges. It follows from $\Sigma s=0$ that $s \otimes T M$ is large only near edges.

On the other hand, the clean map CM of the smoothed deconvolution obeys

$(a+\lambda s) \otimes C M=D M-r$

where $r$ is the residual map. Subtraction from Eq. (10) gives

$a \otimes(T M-C M)=r+\lambda s \otimes C M$

where $T M-C M$ also is large only near edges. The right side being known, we can use Eq. (13) - not to properly solve it; it is too ill-conditioned - but to estimate the maxima of $T M-C M$ and thus $T M$, or the edge height, near the edges. The corresponding estimate of $\lambda s \otimes T M$, calculated only near suspected edges, is added to $r$, and the deconvolution with the modified beam $a+\lambda s$ is continued.

If the solution is known, as in the test examples of the next section, a perfect compensation is possible. We have done this in Figs. 3a and 3e for the square in order to show the Gibbs ringing of the point sources more clearly. The changes need not be large. For instance, the data of the DM (Fig. 1e) have to be changed only near the edges of Fig. 1a but nowhere by more than $0.3 \%$ (Fig. 1f). Note that these changes of the DM are enlarged, in Fig. 1f, by a factor 100 .

In practice, one does not know the edges or point sources and can, thus, only estimate positions and heights of these from preliminary results of a smoothed deconvolution. The criterion for successful steepening is the decrease of the Gibbs phenomenon. We compensate for point sources interactively (example: the point source in Sect. 6). Edges are more difficult. We do not yet have a reliable code that finds all edges correctly and does not invent spurious edges. In simple cases, as the square of Sect. 5, our automatic code works sufficiently well (example: Fig. 2e).

\section{Tests}

We demonstrate the deconvolution capabilities of MIM as well as the Gibbs phenomena by a test square of $40 \times 40$ pixels, with constant intensity inside and zero outside (frame size $128 \times 128$ pixels). The dirty map was constructed by convolving this model with a given VLBI beam (taken from the data described in sec.6). The size of the main beam and, thus, of the smoothing beam is $9 \times 9$ pixels (Fig. 1d). The input data are given in Figs. 1a-f. Note that the maximum height of the DM is more than 300 times the height of what a single point source in Fig. 1a would give. That is, in the center of the DM, only about $0.3 \%$ of the intensity come from emission at that point (Fig. 1f).

Results are given in Fig. 2. The left sides show the inner $40 \times 40$ pixels. The right sides give intensity histograms of the whole map in units of $\%$ of the expected result within the square. The correct result would be $1600 \times 100 \%$ and $14784 \times 0 \%$. With full smoothing (Fig. 2a), the resulting Gibbs phenomenon parallel to the edges amounts to less than $\pm 15 \%$ in this particular case (width of the right spike in Fig. 2b). These high intensities cover the inner $38 \times 38$ pixels of the map (no intensity below $84 \%$ ). The other spikes in Fig. $2 \mathrm{~b}$ at $65 \%$ (the edge line of 156 pixels at the inner side of the circumference of the square which should have $100 \%$ in a perfect deconvolution) and at $30 \%$ (the adjacent outer line of 164 pixels which should be empty in a perfect deconvolution) stem from the smoothing of the edges. The rest of the frame (14620 pixels) is essentially empty and contains less than $0.3 \%$ of the total intensity.

Comparison with the Richardson-Lucy method (RL; Lucy 1974; Hook \& Lucy 1992) as contained in the Nov94 version of MIDAS: RL needs a non-negative beam. We constructed it by setting negative values of the beam (Fig. 1c) to zero, and used a correspondingly changed DM. Also, due to the true Fourier transforms used, RL needs a rather large DM (we used $512 \times 512$ pixels) in order to avoid aliasing. In other words, the deconvolved map must be large enough to be essentially empty. Then the result (Figs. 2c,d) is similar to our fully smoothed result.

That the Gibbs phenomenon can be reduced by automatic edge detection and corresponding correction of the dirty map is shown in Figs. 2e+f. As already mentioned, our detection code is, at present, not reliable enough to report on further details.

Another test was to put, in the center of Fig. 1a, a point source of height 30000 on top of the square with height 300 . That is, the point source has an intensity of $1 / 16$ of the total intensity of the square. Figure 3 gives some MIM results. The circular Gibbs phenomenon due 

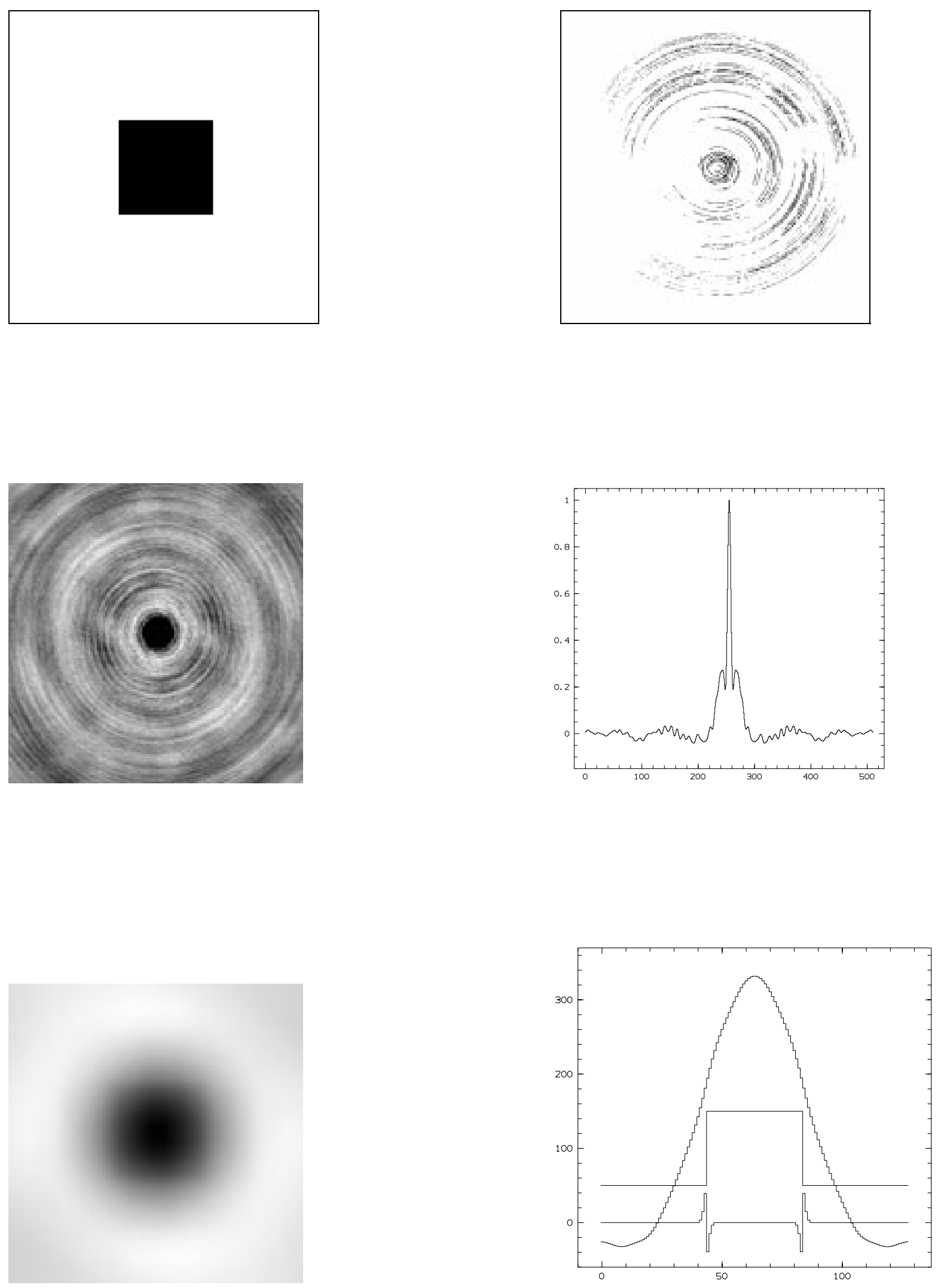

Fig. 1. (a,b): top; e,f) bottom): Deconvolution of a square $(40 \times 40$ pixels in a $128 \times 128$ frame). a) The model. b) $u v$ coverage of the beam. c) Dirty beam ( 4 times larger: $512 \times 512$ pixels). d) Central cross section through beam. e) Dirty map (128 $\times$ $128)$ = model a) convolved with DB c). f) Central cross section through DM e). Also, central cross section through model a), enlarged by a factor of 100 and shifted by 50 units. Finally, cross section through edge correction for DM, again multiplied by a factor 100 

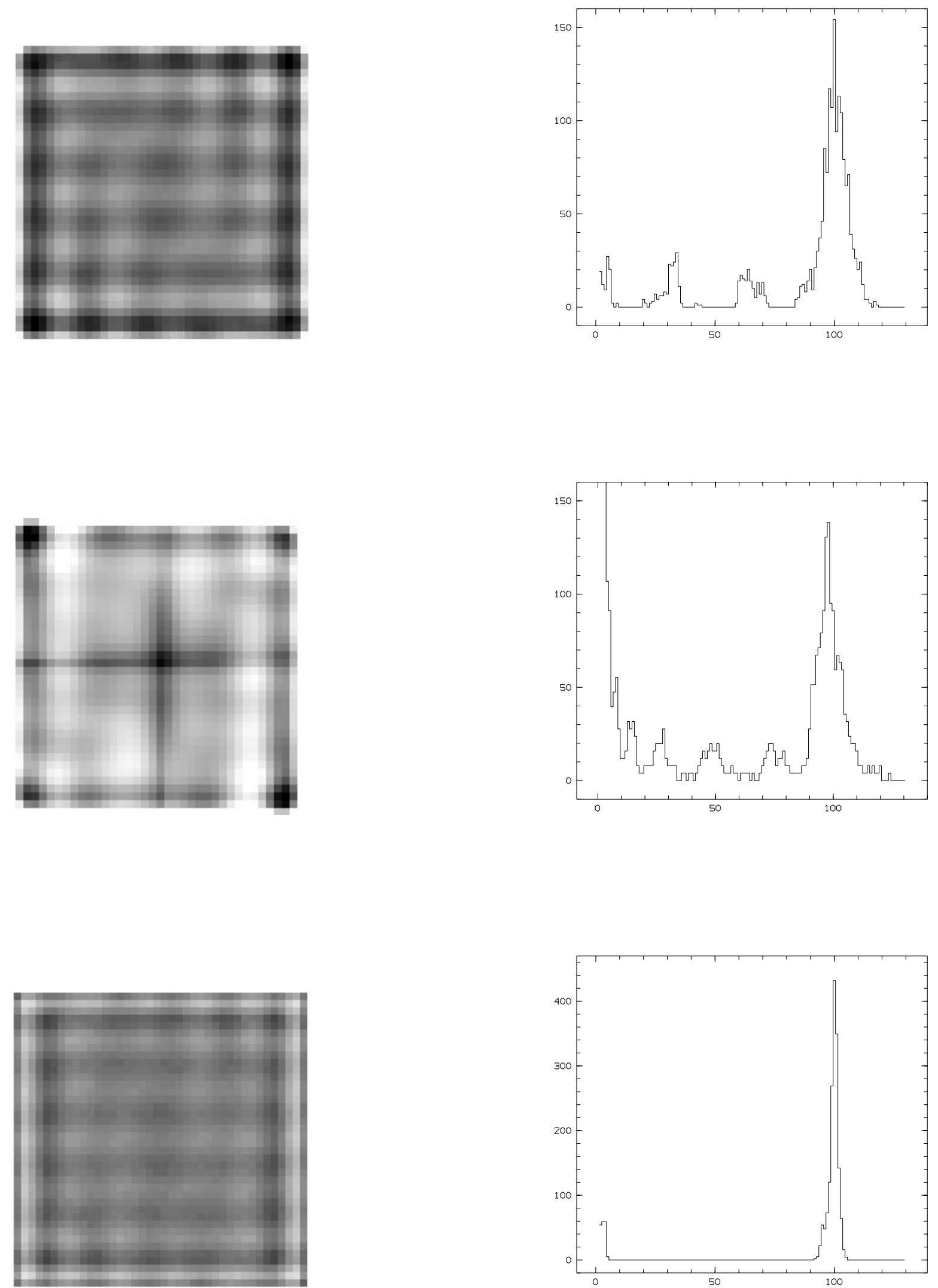

Fig. 2. Results of deconvolution of the square (inner parts of maps, $40 \times 40$ pixels), and intensity histograms in units of percent of correct result in square. The correct result would be a flat square without modulation, and, in the histograms, 1600 times $100 \%$ and $0 \%$ for all the rest of the pixels). a,b) MIM. c,d) Richardson-Lucy 50 iterations (with slightly changed DM and DB). $\mathbf{e , f}$ : MIM with automatic edge correction 

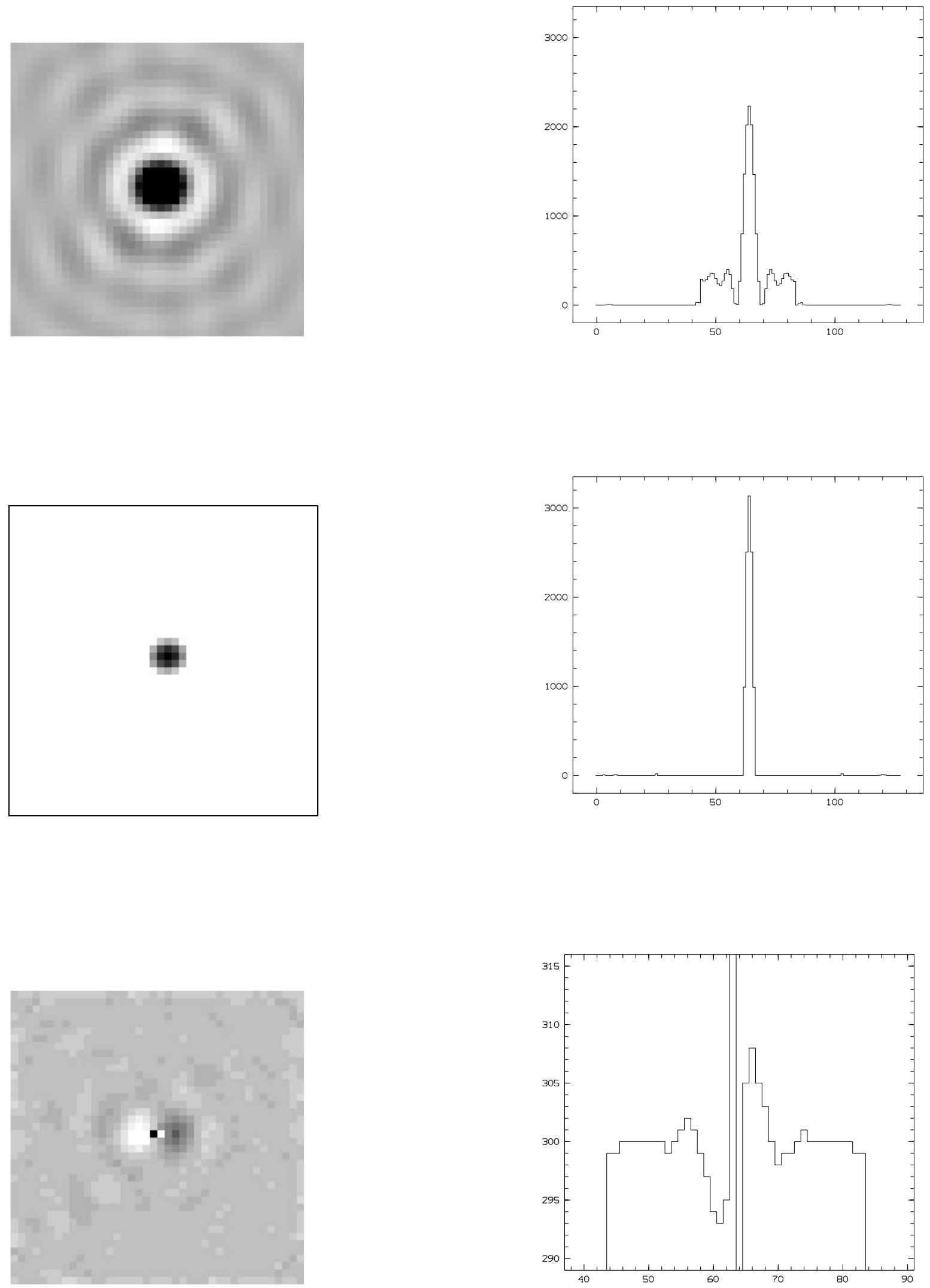

Fig. 3. Point source results: Inner parts $(40 \times 40$ pixels $)$ and central cross sections of deconvolved maps. a,b) Point source (intensity 30 000) on top of extended emission (intensity 300). Gibbs correction for the square, i.e., the remaining Gibbs phenomenon is due to the point source only. c,d) Same point source without underlying square (isolated point source). The Gibbs phenomenon has completely vanished. The reason is the non-negativity constraint in the deconvolution which effectively suppresses the first negative ring and, thus, all outer positive rings. Also, the width is definitely narrower than in a). e,f) Point source (intensity 300) on top of square (intensity 300). "Correct" Gibbs correction but, for the point source, at a position that is shifted by 1 pixel from the correct one 

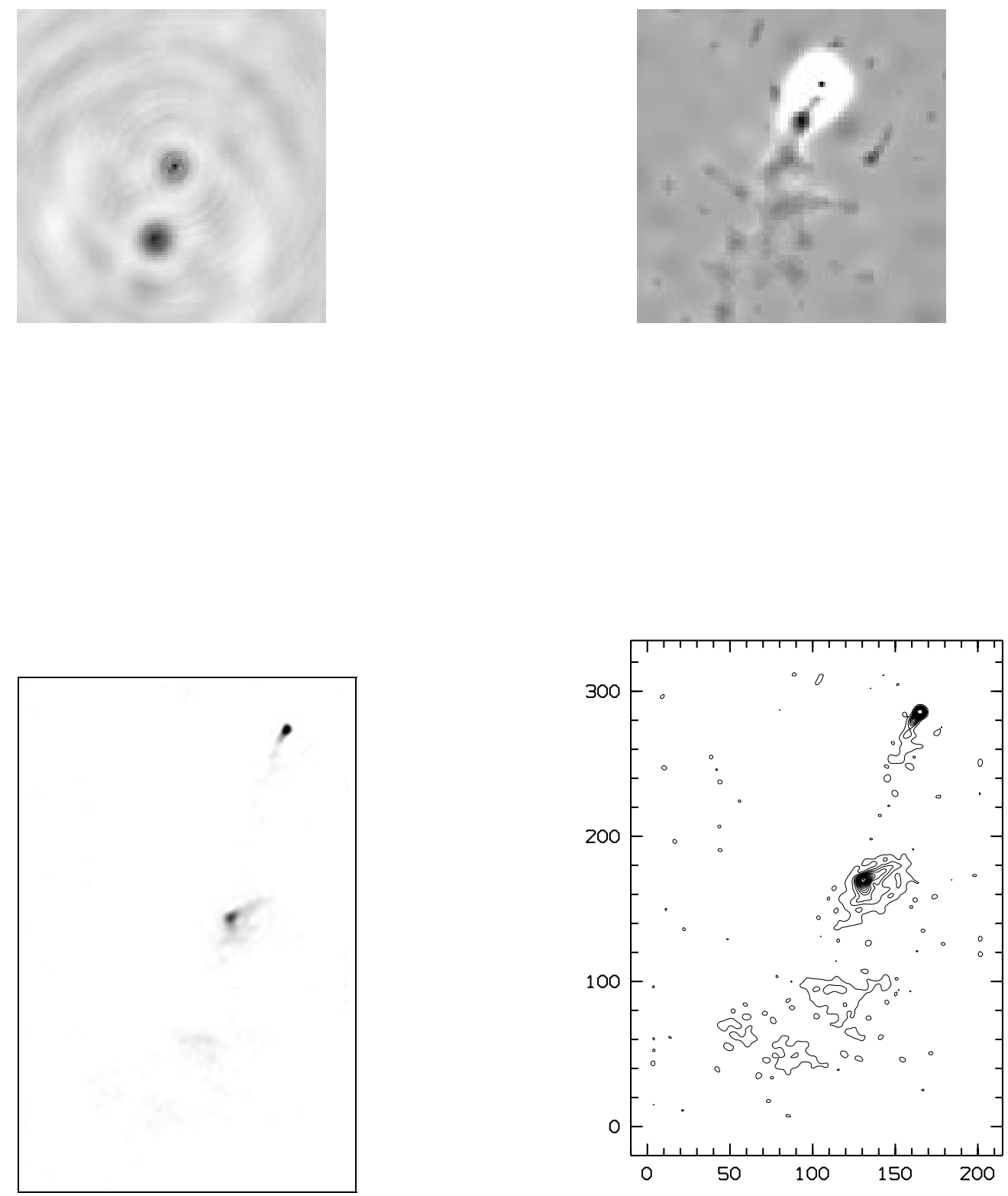

Fig. 4. Results on 3C 309.1. a) DM. b) Difference image of central source and jet (MIM minus CLEAN - see text). The image is enlarged, as compared to $\mathbf{c}$ ) and $\mathbf{d}$ ), by a factor of 3.2. The dark regions (MIM is brighter) are surrounded by brighter rims (here CLEAN is brighter), indicating the better resolution of MIM. c) MIM, 0.2 mas pixel size (map is $316 \times 206$ pixels). North is top, east is left. d) Contour plot of same (levels $2.5 \mathrm{e}-4+2.5 \mathrm{e}-3 \times n, n=0,1, .$. , in units of the central point source)

to the point source is quite drastic. The first negative ring reaches zero intensity. Such Gibbs phenomena are strongly suppressed (Figs. 3c,d) for a point source that is isolated rather than situated on top of an extended source if, as in most deconvolution routines, the result (= intensities) is forced to be non-negative. Also, the point source appears much narrower in Fig. 3d than in Fig. 3b.

Interesting is the superposition of a positive and a negative Gibbs phenomenon if the assumed position of the point source where smoothing is suppressed is chosen wrong by one pixel (Figs. 3e,3f).

\section{Example: 3C 309.1}

Dr. A. Kus of Torun kindly supplied us with VLBI visibilities of $3 \mathrm{C} 309.1$. The observations were done on 08/06/1986 at $5 \mathrm{GHz}$, involving the 12 telescopes Onsala, Medicina, Effelsberg, WSRT, Jodrell Bank, Haystack, 
NRL, NRAO $42 \mathrm{~m}$, VLA E8, OVRO, Hat Creek, and Torun.

A total of $18870 \mathrm{CVs}$ were provided. The $u, v$ coverage is shown in Fig. 1b. The smallest frequency is about 0.8 , the largest $14.610^{7} \lambda$. Due to the lack of small frequencies, all features larger than about $1 / f_{\min }=25$ mas are lost. That means that only the fine structure near the central compact source (Wilkinson et al. 1984) can be seen. The natural beam size is about $1 / f_{\max }=1.7$ mas. It follows that a reasonable pixel size should be between about .2 and .8 mas. The DM (Fig. 4a), the DB (Figs. 1c,d) and the deconvolution result as given in Figs. $4 \mathrm{c}+\mathrm{d}$ refer to a 0.2 mas pixel size. The central source (top right) is unresolved. It must be deconvolved with removed smoothing in order to avoid apparent Gibbs phenomena. It is surrounded by a roughly circular extended emission with half power width of about 1.0 mas and a total width of about 2.0 mas, the center of which is slightly shifted in the direction of the adjacent jet by about 0.1 to 0.2 mas. The southern part of the jet is quite faint and appears to contain indications of weak remaining Gibbs rings caused by the strong central source. A second source near the center of Fig. 4c is barely resolved in the source center and has surrounding extended emission. The brightest pixel is 6.8 mas east and 23.2 mas south of the central point source. A third source (upper bottom) is smoothly extended. The fourth one (lower bottom) has very low level intensity that tends to look knotty but this appears to be an artifact of the deconvolution.

The intensities (in units of the point source which has about $0.2 \mathrm{Jy}$ ) are approximately as follows:

Table 1. Maximum intensities

$\begin{aligned} \text { central point source } & 1.0 \\ \text { central extended source: maximum pixel } & 0.08 \\ \text { second source: maximum pixel } & 0.04 \\ \text { third source: maximum pixel } & 0.004 \\ \text { fourth source: maximum pixel } & 0.001\end{aligned}$

Table 2. Extended intensities

$\begin{array}{rrrr}\text { central source: inner part } & 1.2 \text { within about } & 1 \mathrm{mas}^{2} \\ \text { total } & 1.8 \text { within about } & 3 \mathrm{mas}^{2} \\ \text { jet (total) } & 0.7 \text { within about } & 8 \mathrm{mas}^{2} \\ \text { second source: center } & 0.8 \text { within about } & 1 \mathrm{mas}^{2} \\ & 2.9 \text { within about } & 10 \mathrm{mas}^{2} \\ & 3.9 \text { within about } & 20 \mathrm{mas}^{2} \\ \text { total } & 5.9 \text { within about } & 100 \mathrm{mas}^{2} \\ \text { third source: total } & 2.0 \text { within about } & 40 \mathrm{mas}^{2} \\ \text { fourth source: total } & 1.7 \text { within about } & 90 \mathrm{mas}^{2}\end{array}$

Not much is gained by still smaller pixels of 0.1 mas (not shown) except that the central source still does not show any sign of being resolved. Much larger pixels (2 mas, also not shown) will force the central point source to be smeared out over more than 1 beam which implies the appearance of strong Gibbs rings. Dr. Kus also supplied us with an 0.1 mas pixel size deconvolution of the same data with a modified CLEAN (Kus et al. 1990). The two maps are remarkably similar. However, the final smoothing with the main beam, necessary in CLEAN, results in a slightly decreased resolution. This is, of course, particularly obvious near the central source but is also noticeable in the deconvolution of the jet, and the brightest part of the second source. We show in Fig. $4 \mathrm{~b}$ a part of the difference map (MIM minus CLEAN). The inner parts of the sources appear dark (MIM is brighter) and are surrounded by regions where MIM is fainter, a clear sign of the better resolution by MIM. While the details of the jet appear similar in both deconvolutions, some of them might nevertheless be not more than artifacts common to both methods.

Acknowledgements. We thank Andrzej Kus for stimulating discussions, for giving us his data on 3C 309.1, and for comments on the manuscript. We acknowledge support by the Österreichische Akademie der Wissenschaften (East-West program) and by the Fonds zur Förderung der Wissenschaftlichen Forschung (project P8568).

\section{References}

Cornwell T., 1983, A\&A 121, 281

Gratl H., 1998, PhD Thesis Innsbruck

Hook R.N., Lucy L.B., 1992, in: Science with the Hubble Space Telescope, ESO workshop, p. 245

Kus A.J., Pearson T.J., Wilkinson P.N., Readhead A.C.S., 1990, in: Parsec-scale radio jets. Proc. NRAO Workshop, Zensus J.A., Pearson T.J. (eds). Cambridge University Press, p. 161

Kus A.J., Köb G., Pfleiderer J., 1992, Astron. Ges. Abstr. Ser. 7, 194

Lucy L.B., 1974, AJ 79, 745

Pfleiderer J., 1985, S. Afr. J. Phys. 8, 73

Pfleiderer J., 1988, A\&A 194, 344

Pfleiderer J., 1989, Naturwiss. 76, 297

Sramek A.R., Schwab F.R., 1986, Imaging, in: Synthesis Imaging, Course Notes from an NRAO Summer School, Socorro (NRAO publ., p. 67)

Wilkinson P.N., Spencer R.E., Readhead A.C.S., Pearson T.J., 1984, in: VLBI and compact radio sources, IAU Symp. 110, Reidel, p. 25 